\title{
Multiple field-induced phases in the frustrated triangular magnet $\mathrm{Cs}_{3} \mathrm{Fe}_{2} \mathrm{Br}_{9}$
}

\author{
D. Brüning $\odot{ }^{1}$ T. Fröhlich, ${ }^{1}$ D. Gorkov, ${ }^{1,2}$ I. Císařová, ${ }^{3}$ Y. Skourski, ${ }^{4}$ L. Rossi, ${ }^{5}$ B. Bryant, ${ }^{5}$ S. Wiedmann,,${ }^{5}$ M. Meven $\odot,{ }^{6,7}$ \\ A. Ushakov, ${ }^{8}$ S. V. Streltsov ${ }^{8},{ }^{8,9}$ D. Khomskii, ${ }^{1}$ P. Becker ${ }^{10},{ }^{10}$ L. Bohatý, ${ }^{10}$ M. Braden, ${ }^{1}$ and T. Lorenz ${ }^{1, *}$ \\ ${ }^{1}$ II. Physikalisches Institut, Universität zu Köln, Zülpicher Straße 77, D-50937 Köln, Germany \\ ${ }^{2}$ Heinz Maier-Leibnitz Zentrum (MLZ), Technische Universität München, Lichtenbergstraße 1, 85748 Garching, Germany \\ ${ }^{3}$ Department of Inorganic Chemistry, Charles University in Prague, Hlavova 2030/8, 12843 Prague 2, Czech Republic \\ ${ }^{4}$ Hochfeld-Magnetlabor Dresden (HLD-EMFL), Helmholtz-Zentrum Dresden-Rossendorf, 01314 Dresden, Germany \\ ${ }^{5}$ High Field Magnet Laboratory (HFML-EMFL) and Institute for Molecules and Materials, Radboud University, \\ 6525 ED Nijmegen, The Netherlands \\ ${ }^{6}$ RWTH Aachen University, Institut für Kristallographie, 52056 Aachen, Germany \\ ${ }^{7}$ Jülich Centre for Neutron Science (JCNS) at Heinz Maier-Leibnitz Zentrum (MLZ), 85747 Garching, Germany \\ ${ }^{8}$ M.N. Mikheev Institute of Metal Physics, Ural Branch, Russian Academy of Sciences, 620137 Ekaterinburg, Russia \\ ${ }^{9}$ Ural Federal University, Mira St. 19, 620002 Ekaterinburg, Russia \\ ${ }^{10}$ Abteilung Kristallographie, Institut für Geologie und Mineralogie, Universität zu Köln, Zülpicher Straße 49b, 50674 Köln, Germany
}

(Received 1 June 2021; revised 24 July 2021; accepted 26 July 2021; published 9 August 2021)

\begin{abstract}
The recently discovered material $\mathrm{Cs}_{3} \mathrm{Fe}_{2} \mathrm{Br}_{9}$ contains $\mathrm{Fe}_{2} \mathrm{Br}_{9}$ bi-octahedra forming triangular layers with hexagonal stacking along the $c$ axis. In contrast to isostructural $\mathrm{Cr}$-based compounds, the zero-field ground state is not a nonmagnetic $S=0$ singlet-dimer state. Instead, the $\mathrm{Fe}_{2} \mathrm{Br}_{9}$ bi-octahedra host semiclassical $S=\frac{5}{2} \mathrm{Fe}^{3+}$ spins with a pronounced easy-axis anisotropy along $c$, and interestingly, the intradimer spins are ordered ferromagnetically. The high degree of magnetic frustration due to (various) competing intradimer and interdimer couplings leads to a surprisingly rich magnetic phase diagram. The zero-field ground state is already reached via an intermediate phase, and the high-field magnetization and thermal expansion data for $H \| c$ identify 10 different ordered phases. Among them are phases with constant magnetization of $\frac{1}{3}$, respectively $\frac{1}{2}$, of the saturation value, and several transitions are strongly hysteretic with pronounced length changes, reflecting strong magnetoelastic coupling.
\end{abstract}

DOI: 10.1103/PhysRevB.104.064418

\section{INTRODUCTION}

Magnetic triangular lattices show a large variety of interesting physics and have been intensively studied [1-5]. For example, the search for spin-liquid candidates caused intense studies of hexagonal and triangular magnetic materials like $\mathrm{Cs}_{2} \mathrm{Cu} X_{4}$ with $X=\mathrm{Cl}, \mathrm{Br}, \mathrm{Na}_{2} \mathrm{IrO}_{3}, \alpha-\mathrm{Li}_{2} \mathrm{IrO}_{3}$, and $\mathrm{Ba}_{3} \mathrm{TiIr}_{2} \mathrm{O}_{9}$ [6-10]. In contrast to the theoretical concept of a spin liquid, all these materials show magnetic order at low temperatures. While in the $5 d$ materials spin-orbit coupling plays an important role, the exchange interactions are typically dominating in $3 d$ transition-metal compounds. In $\mathrm{Cs}_{3} \mathrm{Cr}_{2} X_{9}$ with $X=\mathrm{Cl}, \mathrm{Br}$, which contain a hexagonal arrangement of face-sharing $\mathrm{Cr}_{2} X_{9}$ bi-octahedra, the magnetism is dominated by a strong antiferromagnetic intradimer coupling. This yields a singlet ground state and a field-induced magnetic ordering which can be described as a Bose-Einstein condensation (BEC) of magnons [11,12]. Two examples of triangular magnets with rich phase diagrams are the $S=\frac{1}{2}$ Heisenberg system $\mathrm{Cs}_{2} \mathrm{CuBr}_{4}$ that shows nine field-induced phase transitions and a multitude of fractional magnetization plateaus [13], and the semiclassical $S=\frac{5}{2}$ material

*t1@ph2.uni-koeln.de, lorenz@ph2.uni-koeln.de
$\mathrm{RbFe}\left(\mathrm{MoO}_{4}\right)_{2}$ with five ordered phases [14]. In these triangular lattices, magnetic moments lie within the plane, and the interesting phase diagrams occur for in-plane applied fields. Another example is $\mathrm{CuFeO}_{2}$, where the $\mathrm{Fe}^{3+}$ moments order perpendicular to the triangular planes $[15,16]$.

Recently, the material $\mathrm{Cs}_{3} \mathrm{Fe}_{2} \mathrm{Br}_{9}$ was discovered that is isostructural to $\mathrm{Cs}_{3} \mathrm{Cr}_{2} \mathrm{X}_{9}$ and crystallizes in the hexagonal space group $P 6_{3} / \mathrm{mmc}$ with $a=7.5427(8) \AA$ and $c=$ $18.5849(13) \AA$ [17]. The structure consists of face-sharing octahedra forming $\mathrm{Fe}_{2} \mathrm{Br}_{9}$ bi-octahedra in triangular layers. The shortest $\mathrm{Fe}-\mathrm{Fe}$ distance amounts to $3.585(3) \AA$ within the bi-octahedra. The in-plane $\mathrm{Fe}_{-} \mathrm{Fe}_{p}$ distance within the triangular layers is about twice as large, 7.179(2) $\AA$, and the interlayer $\mathrm{Fe}^{-\mathrm{Fe}_{c}}$ distance of 7.543(1) $\AA$ is slightly larger. Based on a study of powder samples, a band gap of $1.65 \mathrm{eV}$ and antiferromagnetic order at $T_{\mathrm{N}}=13 \mathrm{~K}$ were reported [17]. Here, we present a detailed study of the low-temperature ordered phases of $\mathrm{Cs}_{3} \mathrm{Fe}_{2} \mathrm{Br}_{9}$ single crystals up to the saturation magnetization $M_{\mathrm{S}}$ that is reached at $43 \mathrm{~T}(52 \mathrm{~T})$ for a field parallel (perpendicular) to the $\mathbf{c}$ axis.

\section{EXPERIMENTAL}

Single-crystals were grown from aqueous solutions of $\mathrm{CsCl}$ and $\mathrm{FeBr}_{3}$ in a molar ratio of $\sim 2: 1$ and a surplus of $\mathrm{HBr}$ 

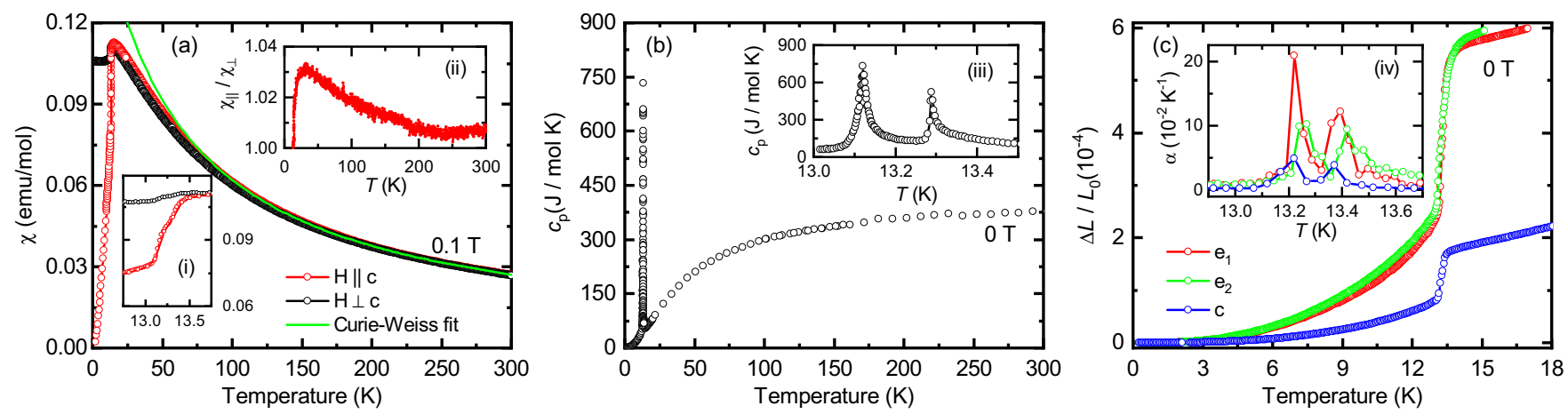

FIG. 1. (a) The temperature dependence of the magnetic susceptibility $\chi_{i}$ for $\mathbf{H} \| \mathbf{c}$ (red) and $\mathbf{H} \perp \mathbf{c}$ (black). The green line stems from a Curie-Weiss analysis of $\chi_{\|}$for $T>100 \mathrm{~K}$. (i) An expanded view around the two-step ordering transition, and (ii) $\chi_{\|} / \chi_{\perp}$ revealing an essentially isotropic susceptibility in the high-temperature paramagnetic phase. (b) The zero-field specific heat with a huge anomaly $\sim 13.2 \mathrm{~K}$ arising from the magnetic order, which actually evolves via a two-step transition with $T_{\mathrm{N} 1}=13.29 \mathrm{~K}$ and $T_{\mathrm{N} 2}=13.12 \mathrm{~K}$, as shown in (iii). (c) The thermal expansion in $0 \mathrm{~T}$ measured along the hexagonal $c$ axis and along two perpendicular directions $e_{1}$ and $e_{2}$ within the $a b$ plane. (iii) The corresponding uniaxial thermal expansion coefficients $\alpha=1 / L_{0} \partial \Delta L_{i} / \partial T$, which again signal the two-step transition.

at room temperature during a period of 6 mo. Further studies revealed that $\mathrm{Cs}_{3} \mathrm{Fe}_{2} \mathrm{Br}_{9}$ can be grown from solutions of $\mathrm{CsBr}$ and $\mathrm{FeBr}_{3}$ in the range of 3:2-4:1 between room temperature and $50{ }^{\circ} \mathrm{C}$.

Using commercial setups [Quantum Design Physical Property Measurement System (PPMS) and Magnetic Property Measurement System (MPMS)], specific heat and magnetic susceptibility were measured between 2 and $300 \mathrm{~K}$, and the low-temperature magnetization was measured up to $14 \mathrm{~T}$. High-field magnetization data were obtained using pickup coils in pulsed magnetic fields up to $56 \mathrm{~T}$ at the high-field center HLD, Dresden Rossendorf. Thermal expansion and magnetostriction $\Delta L(T, H) / L_{0}$ were measured in a homebuilt capacitance dilatometer down to $0.26 \mathrm{~K}$ in magnetic fields up to $17 \mathrm{~T}[18,19]$. The field was applied parallel to the crystal direction whose length change $\Delta L_{i}$ was measured. Here, $L_{0}$ denotes the corresponding overall length of the sample, and the uniaxial thermal expansion coefficient $\alpha=1 / L_{0} \partial \Delta L_{i} / \partial T$ was obtained numerically. Additonally, high-field expansion data up to $37 \mathrm{~T}$ were taken at HFML Nijmegen using commercial dilatometers [20,21].

The crystal structure of $\mathrm{Cs}_{3} \mathrm{Fe}_{2} \mathrm{Br}_{9}$ was investigated via an APEX (Bruker) four-circle single crystal $\mathrm{x}$-ray diffractometer at $150 \mathrm{~K}$ [22]. The low-temperature crystal and magnetic structure was studied on the single-crystal neutron diffractometer HEiDi [23] and on the KOMPASS instrument (both at FRM-II, Munich). On HEiDi, a crystal of 48.680(14) mg was mounted in a way that the $\left(\begin{array}{llll}0 & 1 & 1\end{array}\right)$ direction was oriented along the $\varphi$ axis of the four circle diffractometer, and data were collected with wavelengths $\lambda$ of 1.171 and $0.795 \AA$. On KOMPASS, the measurements were performed in the $(100) /(010)$ and $(100) /(001)$ scattering planes. A polarized beam was obtained through serial polarizing $\mathrm{V}$ shaped multichannel cavities and a highly oriented pyrolytic graphite $[\mathrm{HOPG}(002)]$ monochromator, $\lambda=4 \AA$. An additional $\mathrm{V}$-shaped multichannel cavity was used to analyze the polarization of the scattered beam in the experiments with the second scattering plane yielding a flipping ratio of 11 . Higher order contamination was suppressed with a velocity selector.

\section{RESULTS AND DISCUSSION}

Figure 1(a) shows the temperature dependent magnetic susceptibility for $\mathbf{H} \| \mathbf{c}$ and $\mathbf{H} \perp \mathbf{c}$. In the high-temperature regime; $\chi_{i}$ is isotropic and well described by a Curie-Weiss law. Fixing the spin of the $\mathrm{Fe}^{3+}$ ions to $S=\frac{5}{2}$ the CurieWeiss analysis of $\chi_{\| c}$ yields a Curie temperature $\theta=-56 \mathrm{~K}$ and a reasonable $g$ factor of 2.09 [24]. Below $13 \mathrm{~K}, \chi_{\| c}$ drops to zero, while $\chi_{\perp c}$ remains almost constant, suggesting nearly collinear antiferromagnetic order with moments pointing essentially along c. The magnetic system is weakly frustrated, as is indicated by the frustration factor $|\theta| / T_{\mathrm{N}} \approx$ 4.4. Figure 1(b) shows the specific heat, which reveals a huge anomaly $\sim 13.2 \mathrm{~K}$. As shown in the inset (iii) and discussed in Appendix A 1, we resolve two distinct transitions at $T_{\mathrm{N} 1}=$ $13.29 \mathrm{~K}$ and $T_{\mathrm{N} 2}=13.12 \mathrm{~K}$. Note that the two-step transition is visible in an expanded view of the susceptibility, see inset (i).

Figure 1(c) shows thermal expansion data measured along the hexagonal $\mathbf{c}$ axis and along the two in-plane directions $e_{1}$ and $e_{2}$, which are parallel and perpendicular to the hexagonal in-plane axis a, respectively. At the transition, each dataset shows a steplike relative length change with $\Delta L_{c} / L_{0} \simeq 10^{-4}$ (blue) and $\Delta L_{e_{i}} / L_{0} \simeq 3 \times 10^{-4}$ for both $e_{1} \| \mathbf{a}$ (red) and $e_{2} \perp$ a (green). The enlarged view on the thermal expansion coefficients $\alpha$ in the inset (iv) reveals that each of them also shows two distinct peaks [25]. As will be discussed below, the magnetic transition breaks the threefold in-plane symmetry and allows for three twin domains of orthorhombic symmetry. Thus, a finite magnetoelastic coupling should induce different thermal expansion anomalies of $e_{1}$ and $e_{2}$ in a single-domain sample. However, in the capacitance dilatometer, the crystal is fixed by $\mathrm{CuBe}$ springs, which apply a weak uniaxial pressure along the measured $\Delta L_{i}$ and may cause a partial or full detwinning at the symmetry-breaking phase transition [26,27]. In this case, the $\Delta L_{i} / L_{0}$ measured along $e_{1}$ and $e_{2}$ result from different orientations of twin domains, as is discussed in Appendix A 2.

The crystal structure of $\mathrm{Cs}_{3} \mathrm{Fe}_{2} \mathrm{Br}_{9}$ is displayed in Fig. 2. The fundamental building blocks are $\mathrm{Fe}_{2} \mathrm{Br}_{9}$ bi-octahedra 


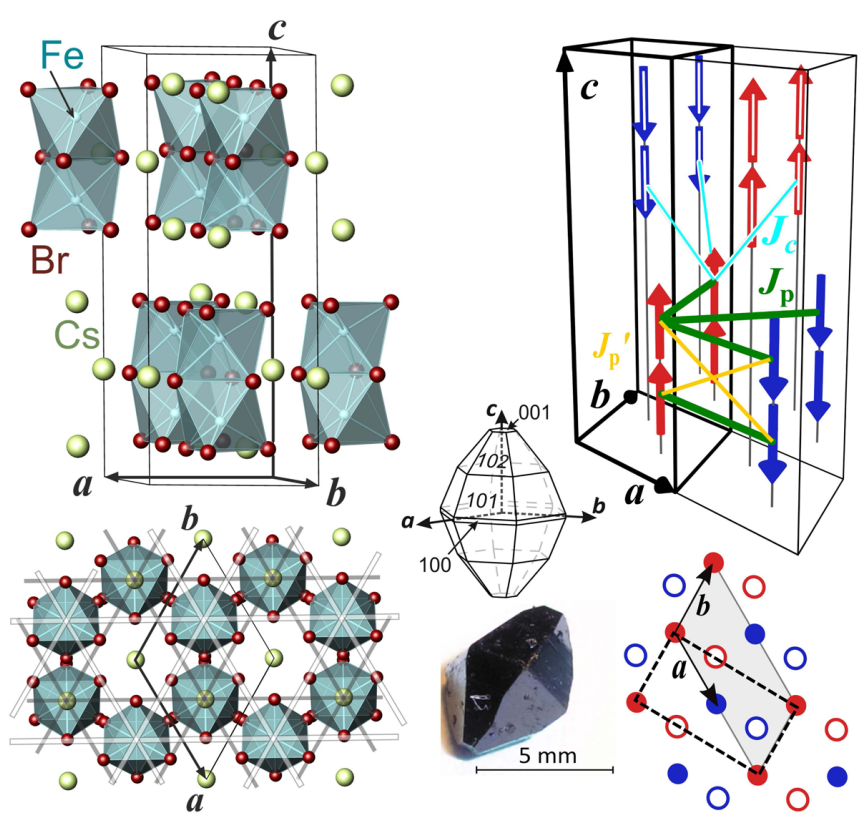

FIG. 2. Crystal (left) and magnetic (right) structure of $\mathrm{Cs}_{3} \mathrm{Fe}_{2} \mathrm{Br}_{9}$. The top panels are three-dimensional (3D) versions, and the bottom panels show the respective projections to the $a b$ plane. In the lower left panel, the solid and open lines connect the $\mathrm{Fe}_{2} \mathrm{Br}_{9}$ bi-octahedra of the lower and the upper triangular planes, respectively. The magnetic structure was determined for the zero-field phase $\mathrm{P} 1$ at $2.5 \mathrm{~K}$, which shows essentially parallel Fe spins within the bi-octahedra. The magnetic unit cell leaves c unchanged, while the hexagonal in-plane axis a is doubled, which results in an orthorhombic unit cell. This is illustrated by the dashed rectangle in the projection to the $a b$ plane, where the open (filled) circles mark Fe ions from the upper (lower) $\mathrm{Fe}_{2} \mathrm{Br}_{9}$ layer, with their magnetic moments pointing either along $+\mathbf{c}$ (red) or $-\mathbf{c}$ (blue). In addition to the intradimer interaction $J$ (not shown), we discuss the interdimer interactions $J_{p}$ (green) and $J_{p}^{\prime}$ (yellow) acting on nearest-neighbor $(\mathrm{NN})$ dimers in the same layer, and the interlayer interaction $J_{c}$ (blue) coupling $\mathrm{NN}$ spins in adjacent dimer layers. The photo shows a single crystal with pronounced facets reflecting the hexagonal structure.

forming triangular planes, which are arranged in the usual $A B A B$ stacking of the hexagonal crystal structure. In the $a b$ projection of the crystal structure (Fig. 2, lower left panel), the bi-octahedra of the different triangular planes are connected by open and solid gray lines. The right panel of Fig. 2 shows the $\mathrm{Fe}^{3+}$ magnetic moments, which are aligned parallel to each other within the $\mathrm{Fe}_{2} \mathrm{Br}_{9}$ dimers for the zero-field ordered phase (see below), and the most important interdimer magnetic exchange interactions are also indicated. Neglecting the interlayer coupling $J_{c}$, the bi-octahedron dimers form frustrated triangular planes which have been long studied [28]. The triangular arrangement is depicted by using open (closed) symbols for the Fe spins of the upper (lower) planes of the three-dimensional (3D) unit cell in Fig. 2. When adding the interlayer coupling $J_{c}$ on an equal footing to $J_{p}$, and when ignoring the intradimer coupling $J$ as well as $J_{p}^{\prime}$, one can also argue about a realization of staggered honeycomb magnetic planes. Note that for antiferromagnetic $J_{p}$ and $J_{c}$ both are frustrated, but $J_{p}$ couples to six neighboring spins of the same triangular plane, while $J_{c}$ couples to three neighbors of the next triangular plane.

The crystal structure was refined in space group $P 6_{3} / \mathrm{mmc}$ at 150,15 , and $2.5 \mathrm{~K}$, yielding no significant differences between the two lowest temperatures and only slight changes in the $\mathrm{Br}$ positions when comparing $150 \mathrm{~K}$ with low-temperature structures, see Appendix B 1. At $2.5 \mathrm{~K}$, i.e., well in the magnetically ordered phase P1, we searched for magnetic Bragg peaks. As is shown in Fig. 3(a), a mapping of the $(h k 0)$ plane in reciprocal space yields additional magnetic Bragg peaks at various half-indexed scattering vectors of type $\left(\frac{n+1}{2}, k, 0\right)$ determining the magnetic propagation vector to $\left(\frac{1}{2}, 0,0\right)$. The star of this propagation vector in the hexagonal structure contains $\left(\frac{1}{2}, 0,0\right),\left(0, \frac{1}{2}, 0\right)$, and $\left(-\frac{1}{2}, \frac{1}{2}, 0\right)$, as can be seen in Fig. 3(a). All observed magnetic Bragg peaks can be indexed with one of these three propagation vectors. We also looked for magnetic Bragg peaks appearing at a half-integer $l$ component but did not observe such intensities. The antiferromagnetic order with these propagation vectors corresponds to the stripe order in a single triangular layer that is illustrated in the lower right part of Fig. 2. The different domains correspond to the propagation vectors and to the stripes rotated by $0^{\circ}, 60^{\circ}$, and $120^{\circ}$.

Temperature-dependent neutron diffraction experiments were performed on HEiDi and on KOMPASS. Figures 3(b) and 3(c) show the amplitudes and the squares of the widths obtained by fitting Gaussian profiles to the scans. The halfindexed magnetic Bragg intensities show only moderate temperature dependence in the ordered phase and abruptly disappear at the lower transition temperature $T_{\mathrm{N} 2}$ in accordance with the first-order character of this phase transition. The scan width only increases above $T_{\mathrm{N} 2}$. To more deeply study the phase transition, additional experiments using polarization analysis were performed on KOMPASS, Figs. 3(d) and 3(e). These experiments reveal sizable diffuse scattering above $T_{\mathrm{N} 2}$ that persists also well above $T_{\mathrm{N} 1}$. At the latter higher transition temperature, we find no signature in the temperature dependence of the diffuse scattering at the halfindexed scattering vector positions. For the two reflections studied $\left(\frac{1}{2}, 0,0\right)$ and $\left(\frac{3}{2}, 0,0\right)$, scans were performed in $h$ and $l$ directions so that the correlations parallel and perpendicular to the planes could be compared. First, the lower transition is not associated with an abrupt increase in the widths, which points to the first-order character and partial coexistence with another ordering scheme in phase P2. Second, there is no indication for a qualitatively distinct behavior when scanning parallel and perpendicular to the planes. In contrast, for a quasi-two-dimensional system, one would expect two-dimensional correlations to survive above the Néel temperature, while the correlation perpendicular to the planes will broaden more rapidly. Therefore, $\mathrm{Cs}_{3} \mathrm{Fe}_{2} \mathrm{Br}_{9}$ is essentially a 3D magnetic system. With the polarization analysis, one may directly determine the orientation of the magnetic moments. We set the neutron polarization axis perpendicular to the scattering plane defined by $(1,0,0)$ and $(0,0,1)$; therefore, the spin-flip (SF) channel at $\left(\frac{1}{2}, 0,0\right)$ and $\left(\frac{3}{2}, 0,0\right)$ records magnetic contributions parallel to $c$, while the non-SF (nSF) channel detects contributions parallel to $b$. At the half-indexed position, there are no nuclear contributions. The data in Figs. 3(f) and $3(\mathrm{~g})$ are corrected for the finite flipping ratio and unambiguously reveal that the magnetic intensities at the half-indexed 

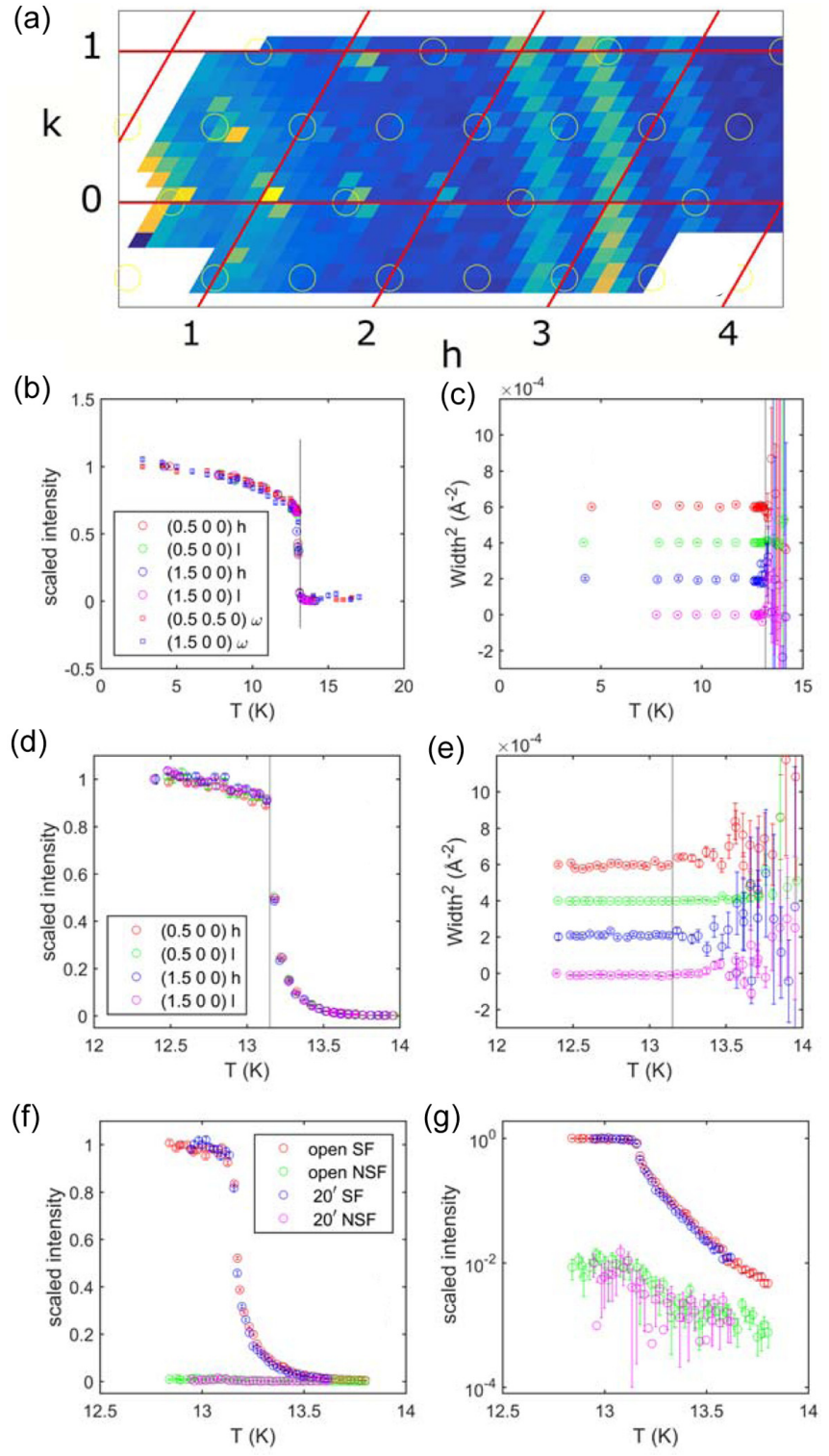

FIG. 3. (a) The neutron diffraction intensity in the reciprocal $(h k l)=(0)$ plane of $\mathrm{Cs}_{3} \mathrm{Fe}_{2} \mathrm{Br}_{9}$ observed on HEiDi at $2.5 \mathrm{~K}$ in zero field. Magnetic superstructure reflections $\left(\frac{3}{2}, 0,0\right),\left(1, \frac{1}{2}, 0\right)$, and $\left(\frac{1}{2}, \frac{1}{2}, 0\right)$ as well as others are clearly visible. Nuclear Bragg reflections exist at the crossings of the red lines, and magnetic peaks are indicated by yellow circles. (b) The peak amplitudes and (c) quadratic widths obtained by fitting Gaussian profiles to the $h, k$ (KOMPASS) or rocking scans (HEiDi) across magnetic peaks. Only for the KOMPASS data, quadratic widths are given after substraction of the low-temperature values (data are vertically shifted for clarity). (d) and (e) The same analysis for data obtained with polarization analysis [spin-flip (SF) with neutron polarization axis along the vertical direction] that focus on the temperature range close to the transition. (f) and (g) The intensities at the magnetic Bragg positions as a function of temperature for SF and non-SF (nSF) channels (measurement with open and $20^{\prime}$ collimation before the sample). All data were taken upon heating.

reflections completely arise from moments pointing along $c$, which agrees with the vanishing magnetic susceptibility $\chi_{\perp c}(T \rightarrow 0)$, Fig $1(\mathrm{a})$. The diffuse scattering visible between
$T_{\mathrm{N} 2}$ and $T_{\mathrm{N} 1}$ and above $T_{\mathrm{N} 1}$ exhibits the same magnetic anisotropy, so that also the short-range correlations are associated with moments along $c$.

The symmetry analysis for the $\left(\frac{1}{2}, 0,0\right)$ propagation vector in space group $P 6_{3} / \mathrm{mmc}$ was performed with the FULLPROF program package [29] and is discussed in Appendix B 2. The four $\mathrm{Fe}$ ions in the primitive cell are all equivalent in the magnetic phase. Furthermore, susceptibility and neutron polarization analysis reveal that the essential part of the magnetic moment aligns along the $c$ direction. Only $\Gamma_{2}, \Gamma_{3}, \Gamma_{6}$, and $\Gamma_{7}$ possess a finite $c$ component, so that the other magnetic models can be excluded. The four irreducible representations correspond to antiferromagnetic or ferromagnetic dimers (two spins in the bi-octahedron) combined with a ferromagnetic or antiferromagnetic stacking within the unit cell. Refinements were performed with these four magnetic structures considering the three domain orientations. The data are only compatible with $\Gamma_{3}$ yielding a weighted $R$ value of $7.7 \%$, while 77,73 , and $80 \%$ are obtained for $\Gamma_{2}, \Gamma_{6}$, and $\Gamma_{7}$, respectively. The parallel alignment of the moments within a dimer can already be deduced from the fact that the strongest magnetic peaks are found in the $(h k 0)$ plane, while for an antiferromagnetic alignment, these intensities exactly cancel (because the two Fe ions exhibit the same $x$ and $y$ coordinates). Also, the antiferromagnetic stacking of the spins within the cell that arises through $J_{c}$ is unambiguous.

The symmetry analysis indicates that the $c$ moments in $\Gamma_{3}$ can be accompanied by an in-plane moment, i.e., a weak canting. Within a dimer, the ferromagnetic $c$ moments are coupled with antiferromagnetic in-plane moments arising from Dzyaloshinski-Moriya interaction. The magnetic refinement with the FULLPROF package only slightly improves with the in-plane moment yielding a total moment of 3.954(5) $\mu_{\mathrm{B}}$, a moment along $c$ of 3.925(5) $\mu_{\mathrm{B}}$, and an in-plane component of $0.47(14) \mu_{\mathrm{B}}$. The three domains occupy similar volume fractions of 39,30 , and $31 \%$. The size of the in-plane moment is consistent with the small reduction of the in-plane susceptibility in the ordered phase. The magnetic structure is illustrated in Fig. 2, neglecting the in-plane component. Here, the spin directions up and down are marked in red and blue, respectively, while spins from the upper (lower) $\mathrm{Fe}^{3+}$ double layer are depicted by open (closed) symbols. Only two-thirds of the in-plane nearest-neighbor (NN) spins show antiparallel orientations, and analogously, only two-thirds of the NN spins across the neighboring planes of the $A B$ stacked bi-octahedra are antiparallel to each other. Thus, all the $\mathrm{NN}$ interdimer couplings $J_{p}, J_{p}^{\prime}$, and $J_{c}$ are geometrically frustrated.

The stripe order described by the $\left(\frac{1}{2}, 0,0\right)$ propagation vector is one possible lowest-energy arrangement of the triangular frustrated magnetic lattice [28]. It breaks rotational symmetry, and the degeneracy can be lifted by magnetoelastic coupling, as it is discussed in Appendix A 2. The magnetic structure and the magnetoelastically distorted structure can be described in the orthorhombic space group Cmcm (No. 63) which results from $P 6_{3} / \mathrm{mmc}$ by breaking the threefold axis. A refinement of the additional structural parameters in the lower space group improves the $R$ values [30] only slightly [31], and a similar improvement can be achieved for the data measured at $15 \mathrm{~K}$, so the structural symmetry reduction induced by the 

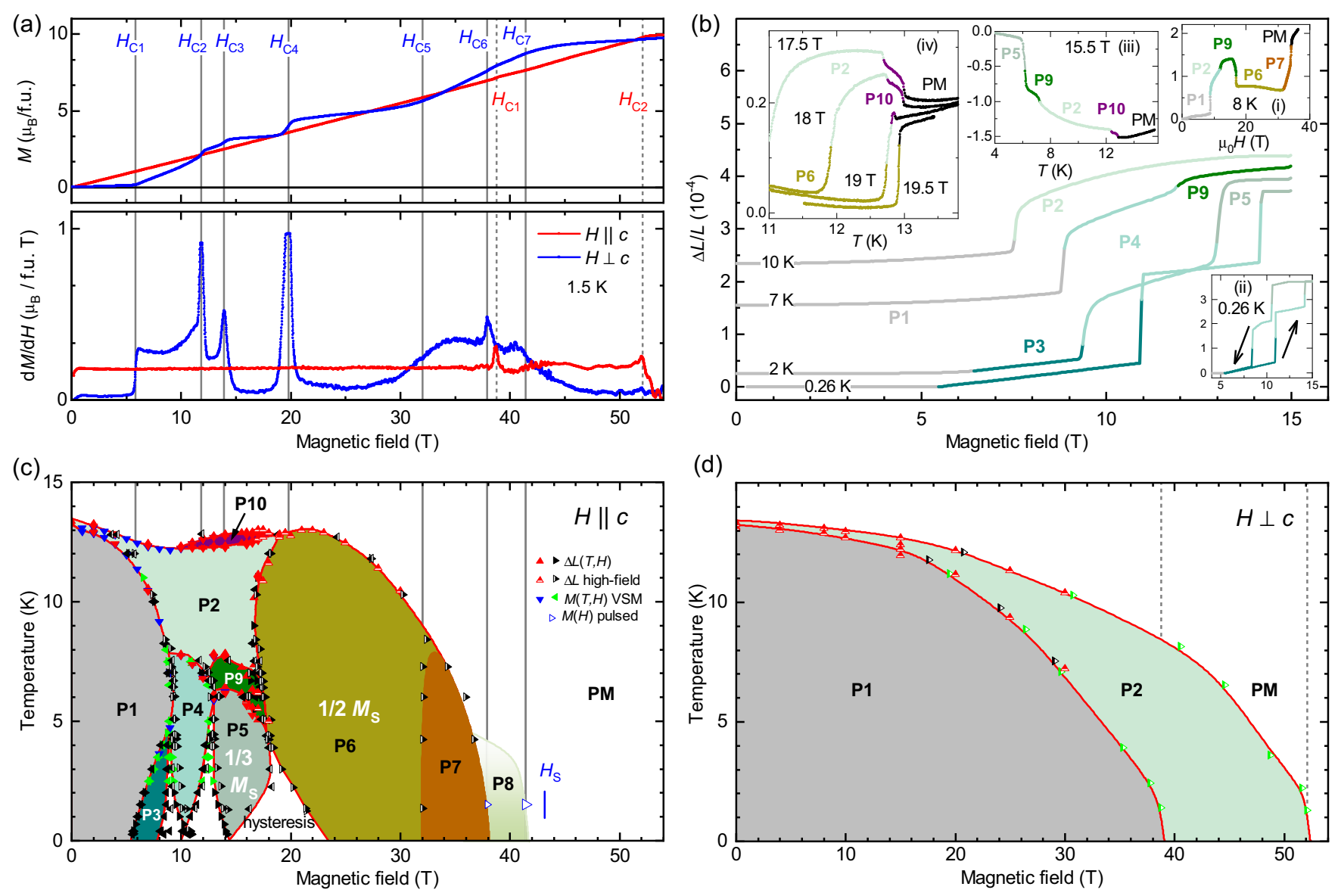

FIG. 4. (a) The pulsed-field magnetization and the differential susceptibility measured at $1.5 \mathrm{~K}$ up to $55 \mathrm{~T}$. The data for $\mathbf{H} \| \mathbf{c}($ blue) reveal seven phase transitions and two transitions for $\mathbf{H} \perp \mathbf{c}$ (red), as marked by the vertical lines. Note that there are two plateau phases (P5 and P6) with constant magnetization of, respectively, $\frac{1}{3}$ and $\frac{1}{2}$ of the saturation $M_{\mathrm{S}}$. (b) Magnetostriction curves $\Delta L_{c}(H) / L_{0}$ obtained with increasing $\mathbf{H} \| \mathbf{c}$ up to $15 \mathrm{~T}$ for representative temperatures from 0.26 to $10 \mathrm{~K}$, and (i) contains an additional curve up to $37 \mathrm{~T}$. The kinks or jumplike changes in $\Delta L_{c}(H)$ signal, respectively, second- or first-order phase transitions with 10 ordered phases (P1 to P10) and the paramagnetic (PM) state. The existence regions of the various phases are marked via the changing colors of $\Delta L_{c}(H, T)$. Strong hysteresis effects occur at $0.26 \mathrm{~K}$, as is shown in (ii). (iii) and (iv) Corresponding thermal-expansion $\Delta L_{c}(T)$ curves in the field range from 15.5 to $19.5 \mathrm{~T}$. (c) and (d) The phase diagrams for $\mathbf{H} \| \mathbf{c}$ and $\mathbf{H} \perp \mathbf{c}$, respectively. Here, the phases are colored as in (b), and the vertical lines mark the critical fields $H_{C 1-7}$ as in (a).

magnetoelastic coupling cannot be resolved in the neutron diffraction study.

As discussed in Appendix $\mathrm{C}$, we mapped the density functional theory plus Hubbard $U$ correction (DFT $+U$ ) total energies of several magnetic configurations onto the Heisenberg model written as $\sum_{i>j} J_{i j} \mathbf{S}_{i} \mathbf{S}_{j}$, and from these calculations the intradimer and the interdimer couplings were determined, see Fig. 2. The intradimer $J$ results from the $\mathrm{Fe}-\mathrm{Br}-\mathrm{Fe}$ exchange paths via the common $\mathrm{Br}$ ions of the face-sharing bi-octahedron with bond angles of $83.5^{\circ}$ and is found to be weakly ferromagnetic with $J=-1$ and $-1.4 \mathrm{~K}$ for $U=5.8$ and $6.5 \mathrm{eV}$, respectively. Interestingly, this coupling is smaller (by absolute magnitude) than the interdimer couplings $J_{p}$ and $J_{c}$, which both result from two $\mathrm{Fe}-\mathrm{Br}-\mathrm{Br}-\mathrm{Fe}$ exchange paths. Here, we obtain values $J_{p}=2.8(3.2) \mathrm{K}$ and $J_{c}=3.0(2.8) \mathrm{K}$ for $U=5.8(6.5) \mathrm{eV}$, whereas for both $U$, a significantly smaller $J_{p}^{\prime}=0.2 \mathrm{~K}$ is obtained for the diagonal interdimer couplings, which result from single $\mathrm{Fe}-\mathrm{Br}-\mathrm{Br}-\mathrm{Fe}$ exchange paths. These parameters are in perfect agreement with the magnetic structure determined at zero field and $2.5 \mathrm{~K}$. Based on these couplings, one can consider $\mathrm{Cs}_{3} \mathrm{Fe}_{2} \mathrm{Br}_{9}$ as a system of frustrated $S=\frac{5}{2}$ triangular layers, which are moderately coupled along c. Due to the hexagonal $A A B B$ stacking of the single $\mathrm{Fe}^{3+}$ layers, the interlayer coupling alternates between the weakly ferromagnetic $J$ for $A A$ and $B B$ and the larger but frustrated antiferromagnetic $J_{c}$ for $A B$. On a meanfield level, the Weiss temperature is given by

$$
\theta=-\sum_{i} \frac{z_{i} J_{i} S(S+1)}{3} \simeq-2.92 \sum_{i} z_{i} J_{i}
$$

with $S=\frac{5}{2}$, and the coordination numbers $z_{i}=1,6,6$, and 3 for the couplings $J, J_{p}, J_{p}^{\prime}$, and $J_{c}$, respectively. Depending on $U$, the $a b$ initio values yield $\theta \simeq-76(-80) \mathrm{K}$. In view of the fact that mean-field theory typically overestimates $|\theta|$ and only near-neighbor couplings are considered, these values agree well with the experimentally observed $\theta \simeq-56 \mathrm{~K}$. Due to the inherent frustration of the triangular arrangement of the $\mathrm{Fe}$ spins, one may expect that relatively small magnetic fields can already induce variations of the magnetic structure.

Pulsed high-field magnetization data of $\mathrm{Cs}_{3} \mathrm{Fe}_{2} \mathrm{Br}_{9}$ at $1.5 \mathrm{~K}$ are shown in Fig. 4(a). For $\mathbf{H} \perp \mathbf{c}, M(H)$ is almost 
linear up to the saturation field of $52 \mathrm{~T}$, and the saturation magnetization $M_{\mathrm{S}}$ of $10 \mu_{\mathrm{B}} /$ f.u. agrees well with two $S=$ $\frac{5}{2} \mathrm{Fe}^{3+}$ ions per formula unit. The differential susceptibility $\chi=\partial M / \partial H$ reveals two peaks at 39 and $52 \mathrm{~T}$, indicating phase transitions. Based on additional data, we derive the phase diagram in Fig. 4(d), containing two ordered phases P1 and P2, which continuously evolve from the two zero-field transitions.

In contrast, multiple transitions occur for $\mathbf{H} \| \mathbf{c}$. Both $\chi_{\|}$and $M(H)$ remain almost zero up to $H_{C 1}=5.6 \mathrm{~T}$, where $M(H)$ starts to increase linearly up to $H_{C 2}=12 \mathrm{~T}$. At $H_{C 2}$, $\chi_{\|}$shows a peak corresponding to a steplike increase of $M\left(H_{C 2}\right)$, followed by another region of constant $\chi_{\|}$. At $H_{C 3}=$ $14 \mathrm{~T}, M$ shows another step to an approximately constant $M\left(H>H_{C 3}\right) \simeq 3.33 \mu_{\mathrm{B}} /$ f.u., which equals $\frac{1}{3}$ of $M_{\mathrm{S}}$. Another steplike increase occurs at $H_{C 4}=20 \mathrm{~T}$, followed by a wide plateau with $M \simeq \frac{1}{2} M_{\mathrm{S}}$ up to $H_{C 5} \approx 32 \mathrm{~T}$. Above this field, $\chi_{\|}$becomes significantly enhanced again up to $H_{C 7} \approx 41.5 \mathrm{~T}$ with an intermediate peak at $H_{C 6}=38 \mathrm{~T}$, and $M_{\mathrm{S}}$ is finally reached above $\sim 43 \mathrm{~T}$. This yields an easy-axis anisotropy $\delta=$ $H_{\mathrm{an}} / H_{\mathrm{ex}}=2\left(H_{\mathrm{S}}^{\perp}-H_{\mathrm{S}}^{\|}\right) /\left(H_{\mathrm{S}}^{\perp}+H_{\mathrm{S}}^{\|}\right) \approx 0.2$, which is a rather large value for a $S=\frac{5}{2}$ material. For comparison, $\delta \approx 0.1$ is found for $\mathrm{RbFe}\left(\mathrm{MoO}_{4}\right)_{2}$ [14], and for $\mathrm{CuFeO}_{2}$, an almost isotropic $\delta \approx 0.02$ is reported [32,33]. Note that, from our $a b$ initio values of the exchange parameters, a saturation field $H_{\mathrm{S}}=4\left[\left(J_{p}+J_{p}^{\prime}\right)+2 J_{c}\right] k_{\mathrm{B}} S / g \mu_{\mathrm{B}} \simeq 33 \mathrm{~T}$ would be expected. This value is below the experimental results for both field directions, indicating that, apart from an anisotropy term, additional exchange couplings between more distant spins should be considered.

Figure 4(b) summarizes representative expansion data $\Delta L_{c}(T, H \| c)$ measured in static fields up to $37 \mathrm{~T}$. At $0.26 \mathrm{~K}$, there is a kink in $\Delta L_{c}\left(H_{C 1}=5.2 \mathrm{~T}\right)$, signaling a second-order phase transition. At $H_{C 2}=11.2 \mathrm{~T}$, the length increases discontinuously by about $1.7 \times 10^{-4}$, followed by another slightly smaller discontinuity at $H_{C 3}=13.2 \mathrm{~T}$. The inset (ii) resolves the pronounced hysteresis between the field-increasing and the field-decreasing run. Because the respective critical fields are shifted by $\sim 3 \mathrm{~T}$, the hysteresis regions of the two field-induced transitions overlap, and consequently, at $\sim 10.6 \mathrm{~T}$ and $0.26 \mathrm{~K}$, each of the three phases can be realized depending on the field-sweep protocol. Typical first-order solid-state transitions are quasidiscontinuous, resulting, e.g., in more or less S-shaped length changes due to a finite transition width and/or phase coexistence. In contrast, the low-temperature transitions in $\mathrm{Cs}_{3} \mathrm{Fe}_{2} \mathrm{Br}_{9}$ are extraordinarily sharp with discontinuous relative length changes of $\sim 10^{-4}$, which systematically change toward continuous variations in $\Delta L_{c}(H)$ upon increasing temperature. The insets (i), (iii), and (iv) display further magnetostriction $\Delta L_{c}(H)$ and thermal expansion $\Delta L_{c}(T)$ measurements, which signal different sequences of field- or temperature-induced magnetoelastic transitions in different regions of the phase diagram.

Combining all anomalies of the thermal-expansion, magnetostriction, and magnetization data reveals the phase diagram in Fig. 4(c). In zero field, there is a two-step transition with an intermediate phase $\mathrm{P} 2$ between the paramagnetic phase and the ground state P1. Below $5 \mathrm{~K}, \mathrm{P} 1$ shows a secondorder transition to $\mathrm{P} 3$ at $5-7 \mathrm{~T}$, which is followed by a cascade of very sharp first-order transitions at $\sim 9,13$, and $20 \mathrm{~T}$ to the phases P4, P5, and P6, respectively. Below $4 \mathrm{~K}$, these first-order transitions become strongly hysteretic. On further increasing field, phase P7 is reached through a second-order transition at $32 \mathrm{~T}$, followed by another first-order transition to $\mathrm{P} 8$ at $\sim 38 \mathrm{~T}$, and $M_{\mathrm{S}}$ is finally reached at $43 \mathrm{~T}$. The phases P1, P5, and P6 are characterized by essentially constant magnetization plateau values of $M=0, \frac{1}{3} M_{\mathrm{S}}$, and $\frac{1}{2} M_{\mathrm{S}}$, respectively, while the other low-temperature phases $\mathrm{P} 3, \mathrm{P} 4, \mathrm{P} 7$, and P8 show more or less linear $M(H)$ behavior with similar slopes $\chi_{\|}$. Above $\sim 7 \mathrm{~K}$ and below $\sim 18 \mathrm{~T}$, three other phases are stabilized. The intermediate phase $\mathrm{P} 2$, which covers only a small temperature interval of $0.2 \mathrm{~K}$ between $T_{\mathrm{N} 1}$ and $T_{\mathrm{N} 2}$ in zero field, continuously grows with increasing field until it finally dominates the intermediate field range from $\sim 8$ to $18 \mathrm{~T}$ at elevated temperature. The additional phases P9 and P10 only form comparatively small pockets. Phase P9 separates P2 from the low-temperature $\frac{1}{2} M_{\mathrm{S}}$ plateau phase P5, and P10 is located between $\mathrm{P} 2$ and the high-temperature paramagnetic phase from 10 to $19 \mathrm{~T}$.

In a first attempt, we consider $\mathrm{Cs}_{3} \mathrm{Fe}_{2} \mathrm{Br}_{9}$ with $S=\frac{5}{2}$ as stacked triangular layers of classical spins, which allows us to compare our data with numerical studies of the field-temperature phase diagram obtained via Monte Carlo simulations [34,35]. The simulations considered triangular layers with antiferromagnetic in-plane $\mathrm{NN}$ and next-NN interactions $J_{1 / 2}$ for a moderate frustration ratio $J_{2} / J_{1}=0.15$ that is sufficient to suppress the so-called $120^{\circ}$ zero-field ground state of pure Heisenberg spins [36]. Along $\mathbf{c}$, a simple $A A$ stacking with ferromagnetic interlayer coupling $J_{\perp} / J_{1}=$ -0.15 was kept constant, while the single-ion anisotropy energy $-D S_{z}^{2}$ was varied from zero up to the strong Ising case $D / J_{1}=1.5$. Interestingly, the simulation for $D / J_{1}=0.5$ [34] reproduces several basic aspects of the experimental phase diagram of $\mathrm{Cs}_{3} \mathrm{Fe}_{2} \mathrm{Br}_{9}$ surprisingly well. The obtained zero-field ground state corresponds to $\mathrm{P} 1$, and a first field-induced transition of second order is expected at $h_{c 1} \simeq h_{S} / 8$, in agreement with the transition from P1 to P3 at $H_{C 1}=5.2 \mathrm{~T}$. Moreover, fractional magnetization-plateau states with $\frac{1}{3} M_{\mathrm{S}}$ and $\frac{1}{2} M_{\mathrm{S}}$ are predicted, which are entered via first-order transitions, as it is observed for the phases P5 and P6. Upon increasing field, the plateau phases are expected to alternate with intermediate states of finite $\chi_{\|}$, which correspond to Bose-Einstein condensates of magnons and are entered via second-order transitions. Based on these numerical results [34], one may suspect the experimentally observed phases $\mathrm{P} 3$ and $\mathrm{P} 7$ to be Bose-Einstein condensates.

The additional phases $\mathrm{P} 2, \mathrm{P} 4, \mathrm{P} 8, \mathrm{P} 9$, and $\mathrm{P} 10$ have no counterparts in the model calculations. Of course, this is not very surprising because the model used in Refs. [34,35] does not capture the specific aspects of $\mathrm{Cs}_{3} \mathrm{Fe}_{2} \mathrm{Br}_{9}$. The $A A B B$ stacking of $\mathrm{Cs}_{3} \mathrm{Fe}_{2} \mathrm{Br}_{9}$ with frustrated antiferromagnetic coupling via $J_{c}$ may induce additional phases. With increasing longitudinal field $\mathbf{H} \| \mathbf{c}$, we can anticipate, e.g., the formation of partial SF phases or incommensurate phases. Indeed, preliminary neutron data indicate that the intermediate zero-field phase P2 is incommensurate, and as shown in Fig. 4, this phase P2 is stabilized for both field directions $\mathbf{H} \| \mathbf{c}$ and $\mathbf{H} \perp \mathbf{c}$, but different microscopic spin structures are expected in longitudinal and transverse fields. Thus, further diffraction studies 
on the field-induced magnetic phases appear very promising to understand this extremely rich phase diagram.

\section{CONCLUSIONS}

In summary, we have identified the material $\mathrm{Cs}_{3} \mathrm{Fe}_{2} \mathrm{Br}_{9}$ as a frustrated triangular antiferromagnet with surprisingly rich properties. The magnetic ordering occurs with a strong magnetoelastic distortion. In contrast to some Cr-based isostructural materials, the spins of the $\mathrm{Fe}_{2} \mathrm{Br}_{9}$ bi-octahedra do not form a dimer singlet ground state but are in fact ferromagnetically aligned. This agrees with our $a b$ initio DFT $+U$ calculations, which yield a weak ferromagnetic intradimer coupling $J$ between the $\mathrm{Fe}$ spins within the $\mathrm{Fe}_{2} \mathrm{Br}_{9}$ bi-octahedra. In contrast, the interdimer coupling $J_{p}$ within the triangular planes is antiferromagnetic and frustrated. A similar antiferromagnetic exchange is obtained for the interlayer coupling $J_{c}$, which acts between spins of neighboring $A B$ layers and is frustrated as well. As a consequence, $\mathrm{Cs}_{3} \mathrm{Fe}_{2} \mathrm{Br}_{9}$ consists of $A A B B$-stacked triangular layers with alternating ferromagnetic and antiferromagnetic coupling along $\mathbf{c}$, which adds to the complexity. The magnetic anisotropy $\delta=0.19$, derived from the saturation fields $H_{\mathrm{S}}^{\perp}$ and $H_{\mathrm{S}}^{\|}$, appears extraordinarily large for spin- $\frac{5}{2}$ moments of the $\mathrm{Fe}^{3+}$ ions with half-filled $3 d$ shells. The strongly different saturation fields are also remarkable because the magnetic susceptibility in the paramagnetic high-temperature phase is essentially isotropic. The origin of the enhanced magnetic anisotropy in the ordered phases is currently unclear. Possibly, it may arise from anisotropic exchange couplings which manifest more strongly in the ordered phases, or the pronounced structural changes upon entering the ordered phase can enhance the single-ion anisotropy. The phase diagram with the magnetic field along the easy axis is very complex and shows a plethora of fieldinduced phases, which include two phases with fractional magnetization plateaus, namely, $\frac{1}{2} M_{\mathrm{S}}$ and $\frac{1}{3} M_{\mathrm{S}}$, and we have indications of at least one incommensurate magnetic phase. Several first-order phase transitions appear with huge hysteresis effects, and sharp lattice deformations occur. All this makes $\mathrm{Cs}_{3} \mathrm{Fe}_{2} \mathrm{Br}_{9}$ an extremely interesting material with very rich and unusual properties.

Note added. While finalizing this paper, we became aware of a very recent publication about the closely related material $\mathrm{Cs}_{3} \mathrm{Fe}_{2} \mathrm{Cl}_{9}$ [37]. The magnetic phase diagrams derived for this isostructural material strongly resemble those of Fig. 4, but with reduced $T_{\mathrm{N}}=5.4 \mathrm{~K}$, reduced saturation fields $H_{\mathrm{S}}^{\|}=$ 19.4 T, $H_{\mathrm{S}}^{\perp}=17.4 \mathrm{~T}$, and smaller anisotropy $\delta=0.1$. Although the proposed interpretation of Ref. [37] concerning the relative importance of various exchange couplings differs from our conclusions, it is gratifying that the basic experimental features of both materials $\mathrm{Cs}_{3} \mathrm{Fe}_{2} \mathrm{Cl}_{9}$ and $\mathrm{Cs}_{3} \mathrm{Fe}_{2} \mathrm{Br}_{9}$ are very similar. This confirms that this extremely rich behavior is indeed an intrinsic property of these materials, although the detailed clarification of the magnetic structures of the different field-induced phases requires further studies.

\section{ACKNOWLEDGMENTS}

We acknowledge support by the German Research Foundation via Project No. 277146847-CRC 1238 (Subprojects A02,
B01, and B04), by the Bundesministerium für Bildung und Forschung, Project No. 05K19PK1, and by the Ministry of Science and Higher Education of Russia via Project Quantum AAAA-A18-118020190095-4. DFT calculations were performed on the Uran supercomputer at the IMM UB RAS. The neutron data were partly taken on the singlecrystal diffractometer HEiDi operated jointly by RWTH Aachen University and JCNS within the JARA collaboration. This paper was supported by HFML-RU/NWO-I and HLDHZDR, members of the European Magnetic Field Laboratory (EMFL).

\section{APPENDIX A: ZERO-FIELD TRANSITIONS}

\section{Hysteresis and magnetic entropy}

As discussed in Refs. [38,39], the usual relaxationtime method used for specific heat measurements in the PPMS is not very applicable for first-order phase transitions. Thus, long heat pulses over a temperature range of $\sim 1 \mathrm{~K}$ were analyzed. As shown in Fig. 5(a), the time-dependent evolution of the sample temperature $T(t)$ has pronounced kinks in both the heating run and in the subsequent relaxation curve, which signal two first-order phase transitions with sharp peaks in the heat capacity. The positions of the respective transition temperatures can be obtained from the derivatives $\partial T / \partial t$, as shown in panel (b). The transition temperatures $T_{\mathrm{N} 1}$ and $T_{\mathrm{N} 2}$ are separated by $170 \mathrm{mK}$, and for both transitions, we observe a small hysteresis of $30 \mathrm{mK}$ between the $T_{\mathrm{N}}$ values obtained with increasing or decreasing temperature.

For the heat capacity data of Fig. 1(b), $c_{p}(T)$ between 13.2 and $13.4 \mathrm{~K}$ was derived from the relaxation curve of Fig. 5 following the procedure described in Ref. [38] and combined with data obtained by the usual relaxation-time method in the remaining temperature ranges. An entropy analysis of these data is presented in Fig. 6. Temperature integration of the measured $c_{p} / T$ data reveals that, despite the rather large $c_{p}$ anomalies at $T_{\mathrm{N} 1}$ and $T_{\mathrm{N} 2}$, the combined entropy release at both transitions is $\sim 4 \mathrm{~J} / \mathrm{molK}$. This corresponds to $13 \%$ of

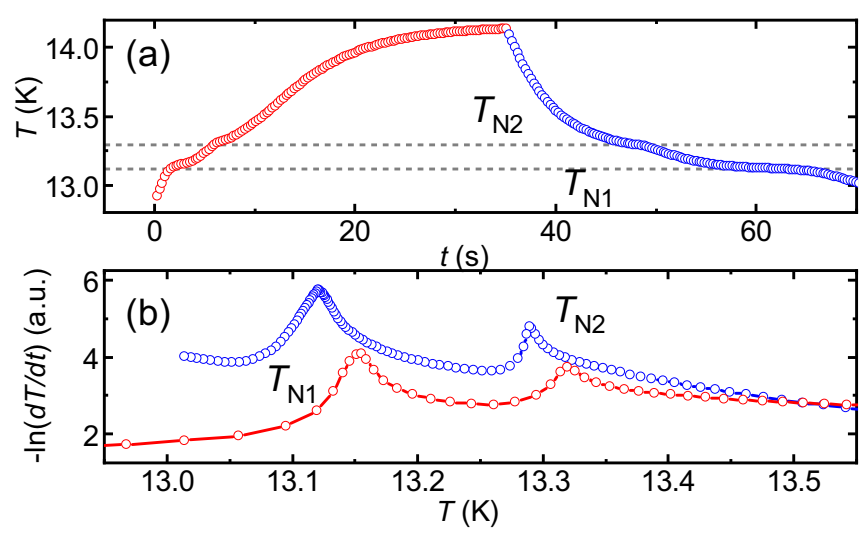

FIG. 5. (a) A long heat pulse in zero field shows two kinks in the time-dependent sample temperature $T(t)$ during both the heating run (red) and in the subsequent relaxation curve (blue). The kinks signal first-order transitions at $T_{\mathrm{N} 1}$ and $T_{\mathrm{N} 2}$. (b) A plot of $-\ln (\partial T / \partial t)$ vs $T$ reveals a weak hysteresis of $\sim 30 \mathrm{mK}$ at each transition. 


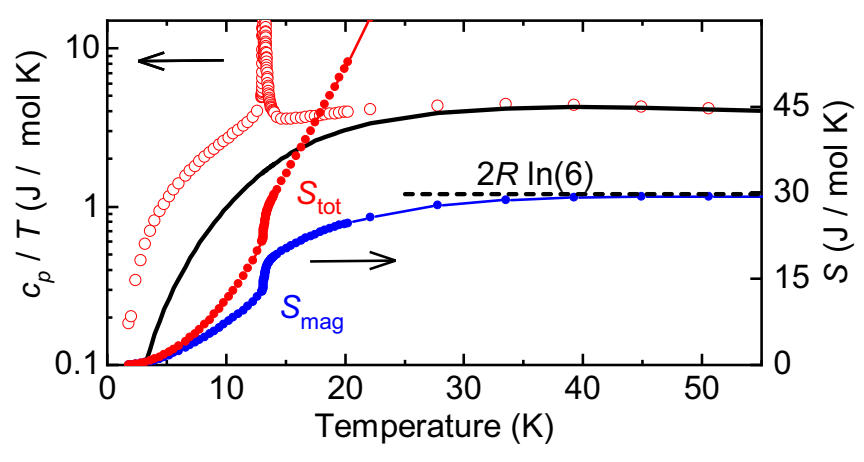

FIG. 6. Heat capacity data (o; left axis) from Fig. 1(b) in a logarithmic plot of $c_{p}^{\text {tot }} / T$ vs $T$ and the corresponding total entropy (red -; right axis) obtained by numerical integration. The solid black line is an estimate of the phononic background $c_{p}^{\mathrm{ph}} / T$ that was adjusted such that the magnetic entropy $S_{\mathrm{mag}}=\int\left(c_{p}^{\mathrm{tot}}-c_{p}^{\mathrm{ph}}\right) / T d T$ (blue) approaches $2 R \ln (2)=29.8 \mathrm{~J} / \mathrm{molK}$, as expected for $\mathrm{Cs}_{3} \mathrm{Fe}_{2} \mathrm{Br}_{9}$.

the full magnetic entropy $2 R \ln (2)=29.8 \mathrm{~J} / \mathrm{molK}$ expected for $\mathrm{Cs}_{3} \mathrm{Fe}_{2} \mathrm{Br}_{9}$ with two $S=\frac{5}{2}$ moments per formula unit. For conventional magnets, most of the magnetic entropy is expected to change continuously below the ordering temperature, but with decreasing (spin and spatial) dimensionality and/or increasing frustration, the continuous magnetic entropy extends toward higher temperature. To analyze this, one has to estimate the phononic background $c_{p}^{\mathrm{ph}}$. Here, we use the solid line in Fig. 6, which comprises a Debye model with additional Einstein modes. The parameters were adjusted such that (i) $c_{p}^{\mathrm{ph}}(T)$ describes the measured data $>$ $\sim 50 \mathrm{~K}$ and (ii) that the expected $S_{\mathrm{mag}}=29.8 \mathrm{~J} / \mathrm{molK}$ is reproduced by $S_{\mathrm{mag}}=\int\left(c_{p}^{\mathrm{tot}}-c_{p}^{\mathrm{ph}}\right) / T d T$. The obtained magnetic entropy release above $T_{\mathrm{N} 1 / 2}$ appears reasonable in view of the moderate frustration ratio $|\theta| / T_{\mathrm{N}} \approx 4.4$ derived from the Curie-Weiss analysis.

\section{Magnetoelastic domains}

Here, we consider the occurrence of magnetoelastic domains resulting from the magnetic order of the zero-field phase P1. As shown in Fig. 2, the in-plane NN spins along one of the three originally equivalent hexagonal a directions are aligned parallel to each other but alternate along the other two a. This results in a twofold in-plane symmetry with the orthorhombic $\mathbf{a}_{o}\left(\mathbf{b}_{o}\right)$ axis along (perpendicular to) the line of parallel spins. For a single-domain crystal, different thermal expansion anomalies are naturally expected for $\mathbf{a}_{o}$ and $\mathbf{b}_{o}$, if there is a finite magnetoelastic coupling. This can be rationalized by assuming that, with the onset of magnetic order, the in-plane bond length of the antiparallel spin pairs weakly shrinks, while the in-plane bond length of the parallel spin pairs weakly increases. For a multidomain crystal, however, the different thermal expansion anomalies of the $\mathbf{a}_{o}$ and $\mathbf{b}_{o}$ axes are expected to cancel each other out, at least partially. Thus, the rather large and essentially identical $\Delta L_{e_{i}} / L_{0}$ measured along the different orientations $e_{1}$ and $e_{2}$ may appear surprising. However, in the used capacitance dilatometer, the crystal is fixed by $\mathrm{CuBe}$ springs, which apply a uniaxial pressure parallel to the measured $\Delta L_{e_{i}}$. This pressure can cause a partial detwinning at the symmetry-breaking phase transition [26,27], and consequently, the almost identical $\Delta L_{e_{i}} / L_{0}$ measured along $e_{1}$ and $e_{2}$ result from different orientations of twin domains. Note that the dilatometer measurements along $e_{1}$ and $e_{2}$ cannot be performed simultaneously.

For a quantitative discussion of the detwinning effects, we consider the variations of the magnetoelastic energy $\partial E_{\mathrm{mag}} / \partial r \propto \delta r$ and of the (nearly) harmonic lattice potential $\partial E_{\text {latt }} / \partial^{2} r \propto \delta r^{2}$ to the lowest order of a generalized lattice distortion $\delta r$. Here, the different powers in $\delta r$ necessarily cause finite lattice distortions to minimize the total energy $E_{\text {mag }}+E_{\text {latt }}$, and these distortions reflect the magnetic symmetry. Consequently, independent spontaneous strains $\delta a_{o}, \delta b_{o}$, and $\delta c_{o}$ along each of the orthorhombic axes are expected for the P1 phase of $\mathrm{Cs}_{3} \mathrm{Fe}_{2} \mathrm{Br}_{9}$. If the variations of $E_{\text {mag }}$ and $E_{\text {latt }}$ are restricted to the respective lowest order in $\delta r$, straightforward geometric considerations reveal that $\delta a_{o}=-\delta b_{o}$. In this case, the hexagonal-to-orthorhombic transition is area conserving with respect to the triangular planes, while $\delta c_{o}$ denotes the bare volume change. Therefore, no sizable inplane length changes should be measured in a fully twinned multidomain sample, which means that the very large and essentially identical contractions $\Delta L_{e_{i}} / L_{0}$ measured along the in-plane directions $e_{1} \perp e_{2}$ result from the shape changes of differently oriented twin domains.

Because $e_{1}$ and $e_{2}$ are, respectively, parallel and perpendicular to one of the hexagonal a axes, these directions correspond to $\mathbf{a}_{o}$ and $\mathbf{b}_{o}$ of one domain and are rotated by $\pm 60^{\circ}$ with respect to the corresponding orthorhombic axes of the other two domains. If we now consider the case that $\mathbf{a}_{o}$ shrinks at the ordering phase transition, the first domain will be favored by uniaxial pressure along $e_{1}$ and a single-domain state can be reached for large enough pressure. For uniaxial pressure along $e_{2}$, the other two domains are favored and a single-domain state cannot be reached. When $n_{1}$ denotes the population of the pressure-induced favored domain, $n_{2}=$ $1-n_{1}$ corresponds to the fraction of the other domains, and the total length changes $\Delta L_{e_{i}} / L_{0}$ measured either along $e_{1}$ or along $e_{2}$ are given by

$$
\begin{gathered}
\delta e_{1}\left(n_{1}\right)=\left(\frac{3}{2} n_{1}-\frac{1}{2}\right) \delta \mathbf{a}_{o}, \\
\delta e_{2}\left(n_{1}\right)=\left(\frac{3}{2} n_{1}-\frac{1}{2}\right) \delta \mathbf{b}_{o}=\left(-\frac{3}{2} n_{1}+\frac{1}{2}\right) \delta \mathbf{a}_{o} .
\end{gathered}
$$

A fully twinned sample has $n_{1}=\frac{1}{3}$, resulting in $\delta e_{1}=\delta e_{2}=$ 0 . By defining a detwinning ratio $r$ that grows from 0 for a fully twinned sample to $r=1$ for a single-domain sample, the above equation can be rewritten as

$$
\begin{gathered}
\delta e_{1}(r)=\left[\frac{3}{2}\left(\frac{1}{3}+\frac{2}{3} r\right)-\frac{1}{2}\right] \delta \mathbf{a}_{o}=r \delta \mathbf{a}_{o}, \\
\delta e_{2}(r)=\left[\frac{3}{2}\left(\frac{1}{3}-\frac{2}{3} r\right)-\frac{1}{2}\right] \delta \mathbf{b}_{o}=-r \delta \mathbf{b}_{o} .
\end{gathered}
$$

From Eqs. (A3) and (A4), it is clear that, for $\delta \mathbf{a}_{o}=-\delta \mathbf{b}_{o}$, the same overall length change can be expected along $e_{1}$ and $e_{2}$ if the uniaxial pressure applied either along $e_{1}$ or $e_{2}$, respectively, results in the same detwinning ratio $r$. However, for pressure along $e_{2}$, a maximum detwinning ratio $r=0.5$ can be reached because two domains are equally favorable. As shown in Fig. 1(c), we observed essentially identical contractions along $e_{1}$ and $e_{2}$, which restricts the detwinning ratio to 
TABLE I. Crystal structure of $\mathrm{Cs}_{3} \mathrm{Fe}_{2} \mathrm{Br}_{9}$ as refined in space group $P 6_{3} / \mathrm{mmc}$ using $\mathrm{x}$-ray data for $150 \mathrm{~K}$ and neutron data for 15 and $2.5 \mathrm{~K}$, respectively. Thermal parameters $U_{i j}$ are given in $\AA^{2}$. The structural refinements with the $150 \mathrm{~K}$ x-ray data were performed with the software package SHELXL-2018/3 [40] and the neutron data with JANA2006 [41]. For Br2 also, $U_{13}=2 U_{23}$ can be finite, and $U_{23}$ was refined to $0.0000(2)$, $-0.0002(3)$, and $0.0011(1) \AA^{2}$ at $2.5,15$, and $150 \mathrm{~K}$, respectively. The lattice parameters amount to $a=7.528(1) \AA$ and $c=18.552(2) \AA$ at $150 \mathrm{~K}$ and to $a=7.491(1) \AA$ and $c=18.477(2) \AA$ at $15 \mathrm{~K}[42]$.

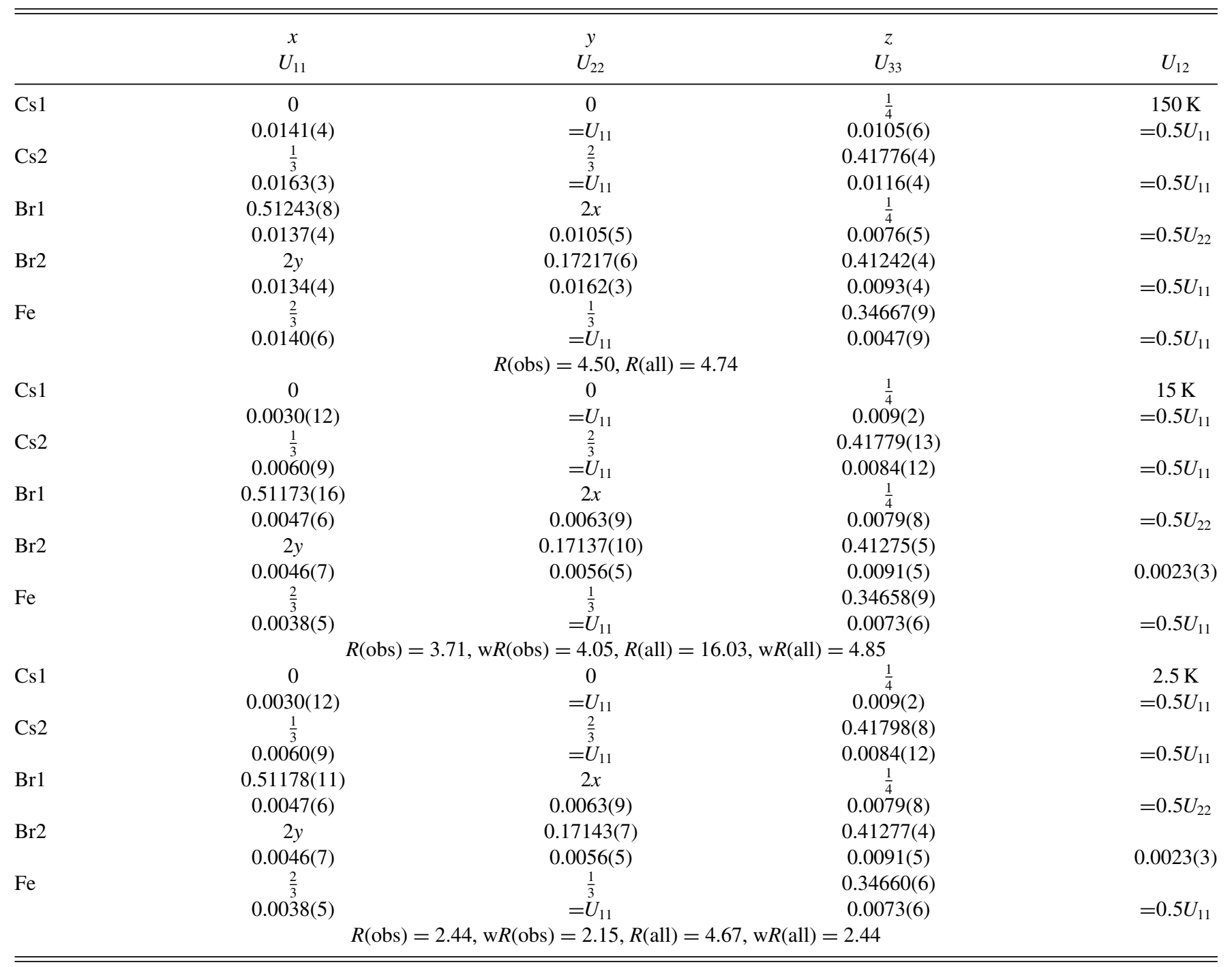

$0<r \leqslant 0.5$, and the intrinsic orthorhombic distortions correspond to $1 / r \Delta L_{e_{i}} / L_{0}$. Using the measured $L_{e_{i}} / L_{0} \simeq 3 \times 10^{-4}$ allows us to give the lower bounds $\left|\delta \mathbf{a}_{o}\right|,\left|\delta \mathbf{b}_{o}\right| \geqslant 6 \times 10^{-4}$, but we cannot judge which of the orthorhombic axes contracts or expands.

\section{APPENDIX B: STRUCTURAL DETAILS}

\section{Crystal structure}

The crystal structure was analyzed at $150 \mathrm{~K}$ using $\mathrm{x}$ rays [22] and at 15 and $2.5 \mathrm{~K}$ with neutrons. On the four-circle neutron diffractometer HEiDi, nuclear reflections were collected using the wavelengths 1.171 and $0.795 \AA$, whereas magnetic reflections were only collected with $\lambda=1.171 \AA$. Reflections that were corrupted by a varying background or by a too-close neighboring Bragg peak were culled manually, so the following numbers refer to the remaining reflections.
For the structural refinement, 1291 reflections were collected at $15 \mathrm{~K}$ (649 of which are unique with respect to space group

TABLE II. Basis vectors of the irreducible representations obtained for the propagation vector $\left(\frac{1}{2}, 0,0\right)$ in space group $P 6_{3} / \mathrm{mmc}$ with four magnetic $\mathrm{Fe}$ ions at $\mathrm{Fe} 1\left(\frac{2}{3}, \frac{1}{3}, 0.35\right), \mathrm{Fe} 2\left(\frac{1}{3}, \frac{2}{3}, 0.85\right), \mathrm{Fe} 3$ $\left(\frac{1}{3}, \frac{2}{3}, 0.65\right)$, and Fe4 $\left(\frac{2}{3}, \frac{1}{3}, 0.15\right)$.

\begin{tabular}{lcccc}
\hline \hline & $\mathrm{Fe} 1$ & $\mathrm{Fe} 2$ & $\mathrm{Fe} 3$ & $\mathrm{Fe} 4$ \\
\hline$\Gamma_{1}$ & $0 \bar{u} 0$ & $0 \bar{u} 0$ & $0 u 0$ & $0 u 0$ \\
$\Gamma_{2}$ & $2 u u v$ & $2 u u \bar{v}$ & $2 u u v$ & $2 u u \bar{v}$ \\
$\Gamma_{3}$ & $2 u u v$ & $2 u u \bar{v}$ & $2 \bar{u} \bar{u} \bar{v}$ & $2 \bar{u} \bar{u} v$ \\
$\Gamma_{4}$ & $0 u 0$ & $0 u 0$ & $0 u 0$ & $0 u 0$ \\
$\Gamma_{5}$ & $0 u 0$ & $0 \bar{u} 0$ & $0 \bar{u} 0$ & $0 u 0$ \\
$\Gamma_{6}$ & $2 u u v$ & $2 \bar{u} \bar{u} v$ & $2 u u v$ & $2 \bar{u} \bar{u} v$ \\
$\Gamma_{7}$ & $2 u u v$ & $2 \bar{u} \bar{u} v$ & $2 \bar{u} \bar{u} \bar{v}$ & $2 u u \bar{v}$ \\
$\Gamma_{8}$ & $0 \bar{u} 0$ & $0 u 0$ & $0 \bar{u} 0$ & $0 u 0$ \\
\hline \hline
\end{tabular}



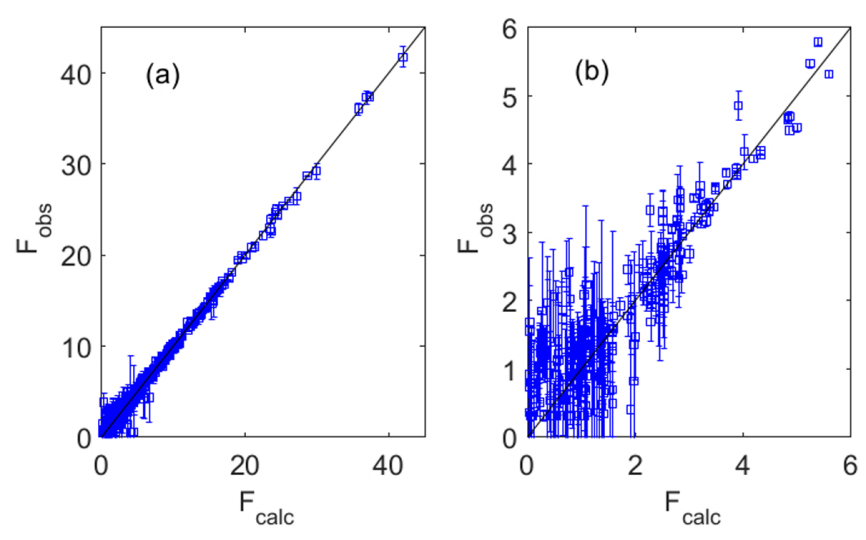

FIG. 7. (a) and (b) The observed structure factors are plotted against the calculated ones for the refinements of the nuclear and magnetic structure with the nuclear and magnetic Bragg reflection intensities, respectively $(T=2.5 \mathrm{~K})$. The FULLPROF program package [29] was used in these refinements.

$P 6_{3} / \mathrm{mmc}$, and 282 unique reflections were observed), and 1109 reflections were collected at $2.5 \mathrm{~K}$ (350 of which are unique, and 272 unique reflections were observed). For the magnetic data collection at $2.5 \mathrm{~K}, 336$ magnetic reflections were collected ( 310 of which belong to different Friedel pairs, and 94 of which were observed).

The structural refinements were performed with the software package SHELXL-2018/3 [40] for the X-ray data taken at $150 \mathrm{~K}$, and with the JANA2006 [41] software package for the low-temperature neutron data. The resulting parameters are given in Table I. The low-temperature structural parameters are identical within the error bars for 15 and $2.5 \mathrm{~K}$, but there are slight differences with the results obtained at $150 \mathrm{~K}$. The largest deviations can be found in the $x$ and $y$ coordinates of the $\mathrm{Br}$ atoms.

\section{Magnetic structure}

The symmetry analysis of the zero-field magnetic structure was performed with the FULLPROF program package [29] and is presented in Table II. Figure 7 presents the comparison of observed and calculated structure factors for the nuclear and magnetic reflections in panels (a) and (b), respectively.

\section{APPENDIX C: DETAILS OF DFT $+\boldsymbol{U}$ CALCULATIONS}

All calculations were performed using the full-potential linearized augmented plane-wave method as realized in the WIEN2K code [43]. We used the Perdew-Burke-Ernzerhof version of the exchange-correlation functional [44]. The supercell used for the total energy calculations consisted of four formula units. The irreducible part of the Brillouin zone was sampled by a $9 \times 5 \times 9 k$-point grid. Strong Coulomb correlations were considered using the DFT $+U$ method [45], and Hubbard onsite electron repulsion and Hund's intraatomic exchange parameters were chosen to be $U=5.8$

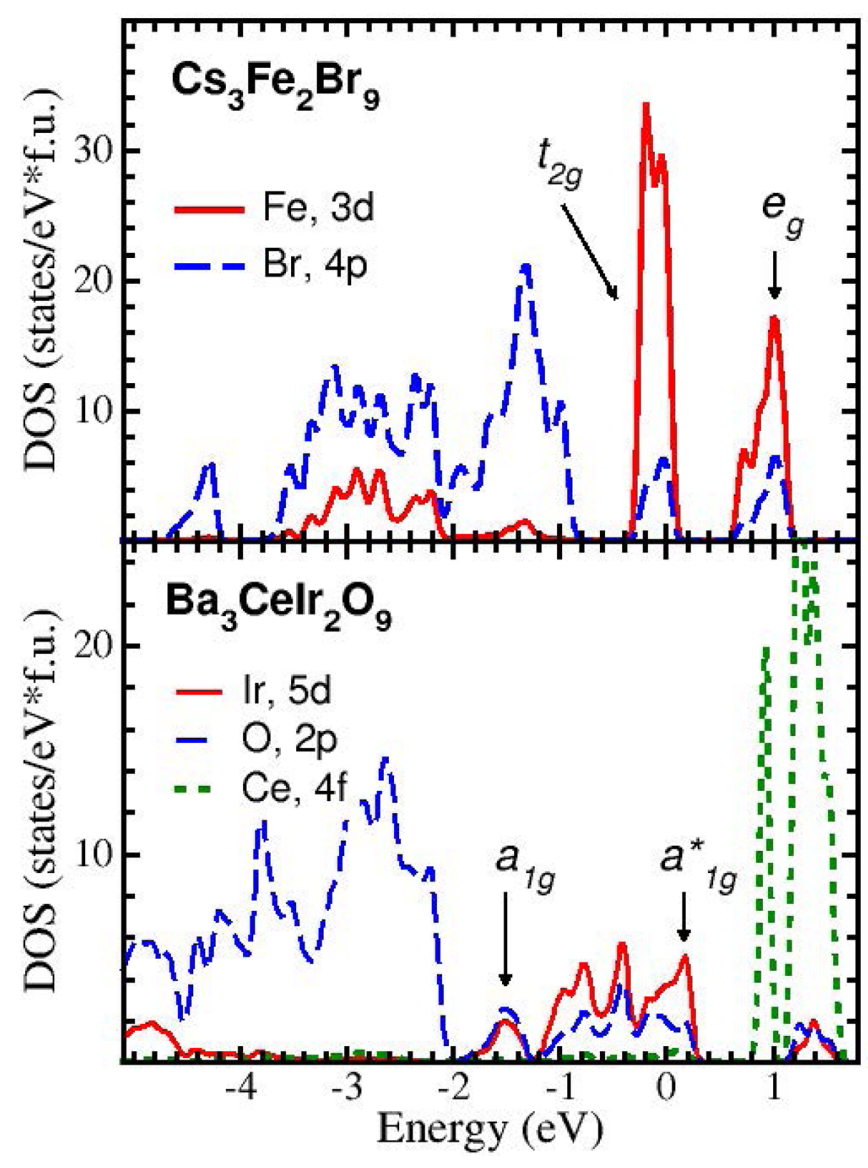

FIG. 8. Partial density of states of $\mathrm{Cs}_{3} \mathrm{Fe}_{2} \mathrm{Br}_{9}$ in comparison with $\mathrm{Ba}_{3} \mathrm{CeIr}_{2} \mathrm{O}_{9}$ (also having structural dimers with face-sharing bi-octahedra) as obtained in nonmagnetic generalized gradient approximation calculations. Molecular Ir-Ir orbitals are seen in the lower panel $\left(a_{1 g}\right.$ marks bonding and $a_{1 g}^{*}$ antibonding states of the corresponding symmetry) in contrast to the ordinary atomic $t_{2 g}$ and $e_{g}$ orbitals in case of $\mathrm{Cs}_{3} \mathrm{Fe}_{2} \mathrm{Br}_{9}$. Fermi energy is zero.

and $6.5 \mathrm{eV}$ and $J_{H}=0.95 \mathrm{eV}$, as for other similar materials [46,47].

The upper panel of Fig. 8 illustrates results of nonmagnetic generalized gradiant approximation calculations of $\mathrm{Cs}_{3} \mathrm{Fe}_{2} \mathrm{Br}_{9}$. One can see that the electronic structure in this case is very different from what we have in another dimer material $\mathrm{Ba}_{3} \mathrm{CeIr}_{2} \mathrm{O}_{9}$ with a very similar crystal structure [48], whose density of states are presented in the lower part of Fig. 8. In $\mathrm{Ba}_{3} \mathrm{CeIr}_{2} \mathrm{O}_{9}$, one clearly sees the formation of the bonding $\left(a_{1 g}\right)$ and antibonding $\left(a_{1 g}^{*}\right)$ bands. There is nothing like this in $\mathrm{Cs}_{3} \mathrm{Fe}_{2} \mathrm{Br}_{9}$, where one may distinguish the atomic $t_{2 g}$ and $e_{g}$ states only. Thus, despite naïve expectations, $\mathrm{Cs}_{3} \mathrm{Fe}_{2} \mathrm{Br}_{9}$ should not be considered a material with molecular orbitals formed by the Fe-3d states, and there is no large exchange coupling between sites as a result. The reason for this is a large ionic radius of $\mathrm{Br}^{1-}(1.96 \AA)$ compared with $\mathrm{O}^{2-}(1.4 \AA)$ [49], which results in a large interatomic distance of $\simeq 3.6 \AA$ between the $\mathrm{Fe}^{3+}$ ions. 
[1] M. F. Collins and O. A. Petrenko, Triangular antiferromagnets, Can. J. Phys. 75, 605 (1997).

[2] H. Kawamura, Spin and chirality orderings of frustrated magnets-stacked-triangular antiferromagnets and spin glasses, Can. J. Phys. 79, 1447 (2001).

[3] R. Moessner, Magnets with strong geometric frustration, Can. J. Phys. 79, 1283 (2001).

[4] O. A. Starykh, Unusual ordered phases of highly frustrated magnets: A review, Rep. Prog. Phys. 78, 052502 (2015).

[5] L. Balents, Spin liquids in frustrated magnets, Nature (London) 464, 199 (2010).

[6] J. Chaloupka, G. Jackeli, and G. Khaliullin, Kitaev-Heisenberg Model on a Honeycomb Lattice: Possible Exotic Phases in Iridium Oxides $A_{2} \mathrm{IrO}_{3}$, Phys. Rev. Lett. 105, 027204 (2010).

[7] S. K. Choi, R. Coldea, A. N. Kolmogorov, T. Lancaster, I. I. Mazin, S. J. Blundell, P. G. Radaelli, Y. Singh, P. Gegenwart, K. R. Choi, S.-W. Cheong, P. J. Baker, C. Stock, and J. Taylor, Spin Waves and Revised Crystal Structure of Honeycomb Iridate $\mathrm{Na}_{2} \mathrm{IrO}_{3}$, Phys. Rev. Lett. 108, 127204 (2012).

[8] S. M. Winter, A. A. Tsirlin, M. Daghofer, J. van den Brink, Y. Singh, P. Gegenwart, and R. Valentí, Models and materials for generalized Kitaev magnetism, J. Phys.: Condens. Matter 29, 493002 (2017).

[9] T. Dey, A. V. Mahajan, P. Khuntia, M. Baenitz, B. Koteswararao, and F. C. Chou, Spin-liquid behavior in $J_{\text {eff }}=$ $\frac{1}{2}$ triangular lattice compound $\mathrm{Ba}_{3} \mathrm{IrTi}_{2} \mathrm{O}_{9}$, Phys. Rev. B 86, 140405(R) (2012).

[10] T. Sakamoto, Y. Doi, and Y. Hinatsu, Crystal structures and magnetic properties of $6 H$-perovskite-type oxides $\mathrm{Ba}_{3} M \mathrm{Ir}_{2} \mathrm{O}_{9}$ $(M=\mathrm{Mg}, \mathrm{Ca}, \mathrm{Sc}, \mathrm{Ti}, \mathrm{Zn}, \mathrm{Sr}, \mathrm{Zr}, \mathrm{Cd}$ and In), J. Solid State Chem. 179, 2595 (2006).

[11] T. Ziman, J. P. Boucher, Y. Inagaki, and Y. Ajiro, Field-induced magnetic order of $\mathrm{Cs}_{3} \mathrm{Cr}_{2} \mathrm{Br}_{9}$ and $\mathrm{Cs}_{3} \mathrm{Cr}_{2} \mathrm{Cl}_{9}$, J. Phys. Soc. Jpn. 74, 119 (2005).

[12] V. Zapf, M. Jaime, and C. D. Batista, Bose-Einstein condensation in quantum magnets, Rev. Mod. Phys. 86, 563 (2014).

[13] N. A. Fortune, S. T. Hannahs, Y. Yoshida, T. E. Sherline, T. Ono, H. Tanaka, and Y. Takano, Cascade of Magnetic-Field-Induced Quantum Phase Transitions in a Spin- $\frac{1}{2}$ Triangular-Lattice Antiferromagnet, Phys. Rev. Lett. 102, 257201 (2009).

[14] A. I. Smirnov, H. Yashiro, S. Kimura, M. Hagiwara, Y. Narumi, K. Kindo, A. Kikkawa, K. Katsumata, A. Y. Shapiro, and L. N. Demianets, Triangular lattice antiferromagnet $\mathrm{RbFe}\left(\mathrm{MoO}_{4}\right)_{2}$ in high magnetic fields, Phys. Rev. B 75, 134412 (2007).

[15] S. Mitsuda, H. Yoshizawa, N. Yaguchi, and M. Mekata, Neutron diffraction study of $\mathrm{CuFeO}_{2}$, J. Phys. Soc. Jpn. 60, 1885 (1991).

[16] D. Inosov, Quantum magnetism in minerals, Adv. Phys. 67, 149 (2018).

[17] F. Wei, F. Brivio, Y. Wu, P. D. Bristowe, and A. K. Cheetham, Synthesis, crystal structure, magnetic and electronic properties of the caesium-based transition metal halide $\mathrm{Cs}_{3} \mathrm{Fe}_{2} \mathrm{Br}_{9}$, J. Mater. Chem. C 6, 3573 (2018).

[18] T. Lorenz, S. Stark, O. Heyer, N. Hollmann, A. Vasiliev, A. Oosawa, and H. Tanaka, Thermodynamics of the coupled spindimer system $\mathrm{TlCuCl}_{3}$ close to a quantum phase transition, J. Magn. Magn. Mater. 316, 291 (2007).

[19] M. Ackermann, D. Brüning, T. Lorenz, P. Becker, and L. Bohatý, Thermodynamic properties of the new multiferroic material $\left(\mathrm{NH}_{4}\right)_{2}\left[\mathrm{FeCl}_{5}\left(\mathrm{H}_{2} \mathrm{O}\right)\right]$, New J. Phys. 15, 123001 (2013).
[20] R. Küchler, T. Bauer, M. Brando, and F. Steglich, A compact and miniaturized high resolution capacitance dilatometer for measuring thermal expansion and magnetostriction, Rev. Sci. Instrum. 83, 095102 (2012).

[21] R. Küchler, A. Wörl, P. Gegenwart, M. Berben, B. Bryant, and S. Wiedmann, The world's smallest capacitive dilatometer, for high-resolution thermal expansion and magnetostriction in high magnetic fields, Rev. Sci. Instrum. 88, 083903 (2017).

[22] The crystallographic data are deposited at the Cambridge Crystallographic Data Centre as supplementary publication, deposition no. CSD 2034800.

[23] M. Meven and A. Sazonov, HEiDi: Single crystal diffractometer at hot source, J. Large-Scale Res. Facil. JLSRF 1, A7 (2015).

[24] The Curie-Weiss analysis of $\chi_{\perp}$ yields essentially the same $g=$ 2.10 but a slightly smaller $\theta=-59 \mathrm{~K}$.

[25] The thermal-expansion anomalies are shifted by $\simeq 100 \mathrm{mK}$ compared with the $c_{p}$ anomalies, which exceeds the typical experimental uncertainties arising from the usage of different samples in different experimental setups. This difference partly arises from the fact that the $c_{p}$ anomalies stem from the longpulse relaxation curves, see Appendix A 1, whereas $\alpha$ was measured upon heating. In addition, a weak upward shift of the transition temperatures can arise due to the uniaxial pressure which is applied when the sample is clamped into the capacitance dilatometer.

[26] S. K. Niesen, G. Kolland, M. Seher, O. Breunig, M. Valldor, M. Braden, B. Grenier, and T. Lorenz, Magnetic phase diagrams, domain switching, and quantum phase transition of the quasione-dimensional Ising-like antiferromagnet $\mathrm{BaCo}_{2} \mathrm{~V}_{2} \mathrm{O}_{8}$, Phys. Rev. B 87, 224413 (2013).

[27] S. K. Niesen, O. Breunig, S. Salm, M. Seher, M. Valldor, P. Warzanowski, and T. Lorenz, Substitution effects on the temperature versus magnetic field phase diagrams of the quasi-one-dimensional effective Ising spin- $\frac{1}{2}$ chain system $\mathrm{BaCo}_{2} \mathrm{~V}_{2} \mathrm{O}_{8}$, Phys. Rev. B 90, 104419 (2014).

[28] G. H. Wannier, The triangular Ising net, Phys. Rev. 79, 357 (1950).

[29] J. Rodríguez-Carvajal, Recent advances in magnetic structure determination by neutron powder diffraction, Phys. B: Condens. Matter 192, 55 (1993).

[30] P. A. Karplus and K. Diederichs, Linking crystallographic model and data quality, Science 336, 1030 (2012).

[31] Improvement by lower space group: From $R$ (obs) $=$ $3.35 \%, \mathrm{w} R(\mathrm{obs})=3.53 \%, R($ all $)=6.15 \%, \mathrm{w} R($ all $)=$ $3.81 \%$ to $R(\mathrm{obs})=3.26 \%, \mathrm{w} R(\mathrm{obs})=3.43 \%, R($ all $)=$ $5.97 \%$, and $\mathrm{w} R($ all $)=3.63 \%$ for the data measured at $2.5 \mathrm{~K}$.

[32] T. T. A. Lummen, C. Strohm, H. Rakoto, A. A. Nugroho, and P. H. M. van Loosdrecht, High-field recovery of the undistorted triangular lattice in the frustrated metamagnet $\mathrm{CuFeO}_{2}$, Phys. Rev. B 80, 012406 (2009).

[33] H. K. Zuo, L. R. Shi, Z. C. Xia, J. W. Huang, B. R. Chen, Z. Jin, M. Wei, Z. W. Ouyang, and G. Cheng, The magnetic anisotropy and complete phase diagram of $\mathrm{CuFeO}_{2}$ measured in a pulsed high magnetic field up to 75 T, Chin. Phys. Lett. 32, 047502 (2015).

[34] L. Seabra and N. Shannon, Supersolid Phases in a Realistic Three-Dimensional Spin Model, Phys. Rev. Lett. 104, 237205 (2010).

[35] L. Seabra and N. Shannon, Competition between supersolid phases and magnetization plateaus in the frustrated easy-axis 
antiferromagnet on a triangular lattice, Phys. Rev. B 83, 134412 (2011).

[36] D. Loison and H. T. Diep, Antiferromagnetic stacked triangular lattices with Heisenberg spins: Phase transition and effect of next-nearest-neighbor interaction, Phys. Rev. B 50, 16453 (1994).

[37] Y. Ishii, Y. Narumi, Y. Matsushita, M. Oda, T. Kida, M. Hagiwara, and H. K. Yoshida, Field-induced successive phase transitions in the $J_{1}-J_{2}$ buckled honeycomb antiferromagnet $\mathrm{Cs}_{3} \mathrm{Fe}_{2} \mathrm{Cl}_{9}$, Phys. Rev. B 103, 104433 (2021).

[38] J. Lashley, M. Hundley, A. Migliori, J. Sarrao, P. Pagliuso, T. Darling, M. Jaime, J. Cooley, W. Hults, L. Morales, D. Thoma, J. Smith, J. Boerio-Goates, B. Woodfield, G. Stewart, R. Fisher, and N. Phillips, Critical examination of heat capacity measurements made on a quantum design physical property measurement system, Cryogenics 43, 369 (2003).

[39] A. Scheie, LongHCPulse: Long-pulse heat capacity on a Quantum Design PPMS, J. Low Temp. Phys. 193, 60 (2018).

[40] G. M. Sheldrick, Crystal structure refinement with SHELXL, Acta Crystallogr. Sect. C 71, 3 (2015).

[41] V. Petrícek, M. Dušek, and L. Palatinus, Crystallographic computing system JANA2006: General features, Z. Kristallogr. 229, 345 (2014).

[42] C. Braschoss, Bachelor thesis, Universität zu Köln, 2019.
[43] P. Blaha, K. Schwarz, G. Madsen, D. Kvasnicka, and J. Luitz, An augmented plane wave + local orbitals program for calculating crystal properties, Techn. Universität Wien, 2001.

[44] J. P. Perdew, K. Burke, and M. Ernzerhof, Generalized Gradient Approximation Made Simple, Phys. Rev. Lett. 77, 3865 (1996).

[45] A. I. Liechtenstein, V. I. Anisimov, and J. Zaanen, Densityfunctional theory and strong interactions: Orbital ordering in mott-hubbard insulators, Phys. Rev. B 52, R5467 (1995).

[46] S. Streltsov, A. Shorikov, S. Skornyakov, and A. Poteryaev, Unexpected 3+ valence of iron in $\mathrm{FeO}_{2}$, a geologically important material lying "in between" oxides and peroxides, Sci. Rep. 7, 13005 (2017).

[47] A. V. Ushakov, A. O. Shorikov, V. I. Anisimov, N. V. Baranov, and S. V. Streltsov, Suppression of magnetism under pressure in FeS: A DFT + DMFT study, Phys. Rev. B 95, 205116 (2017).

[48] A. Revelli, M. Moretti Sala, G. Monaco, P. Becker, L. Bohatý, M. Hermanns, T. C. Koethe, T. Fröhlich, P. Warzanowski, T. Lorenz, S. V. Streltsov, P. H. M. van Loosdrecht, D. I. Khomskii, J. van den Brink, and M. Grüninger, Resonant inelastic x-ray incarnation of Young's double-slit experiment, Sci. Adv. 5, eaav4020 (2019).

[49] R. D. Shannon, Revised effective ionic radii and systematic studies of interatomic distances in halides and chalcogenides, Acta Crystallogr. Sect. A 32, 751 (1976). 